\title{
Melancholie oder mal du siècle und écriture
}

Wenn wir uns mit der Melancholie und deren Beziehungen zur Gesellschaft ${ }^{1}$ wie zum literarischen Schreiben ${ }^{2}$ im Rahmen dieser Vorlesung beschäftigen, so sollten wir dies vorrangig als Philologinnen und Philologen tun. ${ }^{3}$ Die Melancholie ist aus der literaturwissenschaftlichen Perspektive zunächst einmal ein Textelement, das sich nicht von ungefähr schon im großen Bezugstext des „Préromantisme“ und der Empfindsamkeit, Bernardin de Saint-Pierres Paul et Virginie, leicht finden lässt.

Von Beginn an steht dort die Melancholie in einer unmittelbaren textuellen Beziehung zum Körper der von ihr erfassten Romanfiguren. Charakteristisch ist für den Melancholiker oder die Melancholikerin die Haltung des auf eine Hand gestützten Kopfes. Diese melancholische Grundhaltung findet sich an ungezählten Stellen in diesem Roman und betrifft fast alle Mitglieder der kleinen Gemeinschaft unter den Wendekreisen; so etwa die Figur des Erzählers inmitten der Ruinen oder die Sklavin Marie, die von einem Felsen aus aufs offene Meer blickt, wohin Virginie mit ihrer Fregatte verschwunden ist. Vor allem erfasst sie natürlich Paul, der an dieser Krankheit schließlich zugrunde gehen wird. Die Melancholie bringt kein plötzliches Sterben mit sich, sondern ein langsames Siechtum, sozusagen ein Sterben in Zeitlupe, begleitet von zermürbenden und unabschließbaren Grübeleien. Die Melancholie ist eine Krankheit der Reflexion und Selbstreflexion, welche sich in verschiedensten Symptomatiken äußert, die freilich allesamt pathologisch sind.

Seit der Antike ${ }^{4}$ weiß man von dieser Krankheit der schwarzen Ideen. Und als eine Krankheit, die den Körper befällt, sah man diese Symptomatik auch im Übergang zum 19. Jahrhundert, wobei man sie auf Grund ihrer massenhaften Verbreitung bald als „Übel des Jahrhunderts“ apostrophierte, als „mal du siècle“.

1 Die Literatur zum Thema Melancholie und Gesellschaft ist kaum mehr zu überschauen; immer noch hilfreich ist Lepenies, Wolf: Melancholie und Gesellschaft. Frankfurt am Main: suhrkamp 1969.

2 Vgl. Lehnert, Gertrud: Herzanker. Dichterinnen und die Melancholie. Berlin: Aufbau Verlag 2011. 3 VBgl. aus psychoanalytischer Sicht Freud, Sigmund: Trauer und Melancholie (1917). In (ders.): Studienausgabe. Band III: Psychologie des Unbewußten. Frankfurt am Main: Fischer 1989, S. 194212; aus der unendlichen Forschungsliteratur möchte ich hervorheben Starobinski, Jean: Mélancholie im Spiegel. Baudelaire-Lektüren. Aus dem Französischen von Horst Günther. München Wien: Hanser Verlag 1992; sowie Benjamin, Walter: Der Saturnring oder Etwas vom Eisenbau. In (ders.): Das Passagenwerk. Band II. Frankfurt am Main: Suhrkamp 1983, S. 1060-1065; sowie Sontag, Susan: Under the Sign of Saturn. New York: Vintage Books 1981.

4 Einen leichtfüßigen Einstieg in die Materie bietet die Sondernummer „Littérature et mélancholie“ des Magazine littéraire (Paris) 244 (1987), S. 14-56. 
Die Melancholie ist sicherlich auch - wie Victor Hugo später einmal meinte eine sanfte, schleichende Krankheit; auch die Brüder Goncourt sprachen von der „tristesse qui n'est pas sans douceur“, von einer Traurigkeit, die nicht der Süße entbehrt. Es ist eine Süße, die freilich nicht selten tödlich endet, wofür es in den Literaturen der Welt unendlich viele Beispiele gibt.

Einige davon haben wir auf unserem Weg zur europäischen Romantik bereits gesehen. Nicht umsonst überbietet Atala ja Virginie gerade darin, dass sie sich selbst aus dem Leben in den Tod - oder das ,Ewige Leben“ - befördert, getragen von einer abgrundtiefen Melancholie, die noch auf ihrem toten Körper ein allerletztes Mal erscheint. Doch ansteckend ist diese Krankheit auch, ja sie affiziert die unterschiedlichsten Figuren von Atala. Denn die Seuche der Melancholie springt über auf viele andere, natürlich in erster Linie auf Atalas Liebhaber, den ,Wilden“ Chactas. Bei Atala führt diese Krankheit wie schon bei Bernardins Paul zu einer wahren Implosion - just so, wie Sigmund Freud ein gutes Jahrhundert später diese Krankheitsform diagnostizierte. ${ }^{5}$ Nicht zufällig greift die schöne Mestizin zum Pharmakon, zum Gift, zerstört ihren Körper damit von innen und gerade nicht von außen her, etwa durch einen Sprung, ein Erhängen oder einen Schuss. Bis heute sind übrigens die Selbsttötungsarten - wie auch die Variationen des Mordes - zwischen Männern und Frauen sehr ungleich verteilt, doch können wir dieser Tatsache hier nicht weiter nachgehen. Fest steht aber mit Blick auf Chateaubriands Atala, dass die Art ihres Todes, ihres Todeskampfes, noch einmal vor Augen führt, dass es letztlich eine Krankheit von innen heraus war, die Atala in eine literarisch wahrlich unsterbliche Tote verwandelte.

Das breite gesellschaftliche Interesse an der Melancholie, die sich Ende des 18. Jahrhunderts geradezu epidemieartig ausbreitete, war auch Ende des 20. Jahrhunderts recht stark verbreitet. Nicht umsonst sind gerade die achtziger Jahre, aber auch zum Teil noch die Neunziger ein Haupttummelfeld für Literatur - auch kritische Forschungsliteratur - zum Thema Melancholie. Es gibt nicht zuletzt auch eine ganze Reihe von Doktorarbeiten, die sich aus unterschiedlichster Sicht dieser Problematik zuwenden, darunter auch eine Arbeit zu Madame de Staël, mit der wir uns später ein wenig beschäftigen werden. ${ }^{6}$ Ein frühes Zeugnis des damals wachsenden Interesses an dieser Krankheit und Seins-Form war Wolf Lepenies' bereits zitiertes Buch Melancholie und Gesellschaft; charakteristischer noch für die Interessenlage der achtziger Jahre des zurückliegenden Jahrhunderts

5 Vgl. Freud, Sigmund: Trauer und Melancholie (1917). In (ders.): Studienausgabe. Band III: Psychologie des Unbewußten. Frankfurt am Main: Fischer 1989, S. 194-212.

6 Vgl. Amend, Anne: Zwischen „Implosion“ und „Explosion“ - Zur Dynamik der Melancholie im Werk der Germaine de Staël. Trier: Wissenschaftlicher Verlag Trier 1991. 
war dann Julia Kristevas Soleil noir, ${ }^{7}$ das gerade der weiblichen Depression - oder in ihrem Sinne „De-Pression“ - eine besondere psychoanalytisch wie texttheoretisch geschulte Aufmerksamkeit schenkte. So haben nicht nur Epidemien, sondern auch Forschungen über Epidemien ihre jeweiligen Interessenlagen und Konjunkturen.

Eines blieb dabei jeweils klar: Es gibt unterschiedlichste Definitionen von Melancholie aus soziologischer wie medizinischer, aus psychologischer wie psychoanalytischer, aus kulturgeschichtlicher wie texttheoretischer oder literaturwissenschaftlicher Sicht, aber doch keine Definition, die alle Seiten befriedigen könnte. Sören Kierkegaard war es, der die Krankheit nicht als Krankheit der Romantik, sondern als ein Phänomen zwischen Neurose und Wahnsinn bestimmte und damit auf eine tiefe Beziehung zum Schreiben aufmerksam machte, die unverkennbar über den Dichter als Neurotiker verläuft. Die Melancholie besitzt daher eine starke Affinität zum Schreiben, und letzteres kann - wie Literatur insgesamt wiederum stark heilsam, mit heilenden Wirkungen versehen sein.

Melancholie, dies wissen wir spätestens seit Atala, ist jedoch nicht nur das „mal de vivre“, sondern verstärkt auch die „impossibilité de vivre“, also die Unmöglichkeit, überhaupt noch weiter leben zu können. Laut Julia Kristeva kann die Melancholie von der Psychiatrie aus auch als Verlust der Lebenslust bezeichnet und beschrieben werden, wobei deren leichtere Variante auch als Depression benannt werden darf. Kristeva greift in ihren texttheoretisch unterfütterten Überlegungen auch und vor allem auf Freuds aus dem Jahr 1917 stammenden Text Trauer und Melancholie zurück.

Auch mit Blick auf Atala ist die Erkenntnis Kristevas interessant, dass es sich um ein bei Frauen häufigeres Phänomen handle, das man landläufig auch mit „la déprime“ bezeichnet, und dass diese Art weiblicher Depression häufig mit einer problematischen Mutterbeziehung einhergehe. Dies kann man in Hinblick auf Atalas Lebensgeschichte nun wirklich sagen, ist ihre Beziehung zur Mutter doch auf Grund des Schwurs ihr gegenüber, sich nie auf einen Mann einzulassen, gerade das lustvernichtende Prinzip schlechthin, dem Atala selbst, aber auch Chactas schließlich auch erliegen werden. Atala ist die Frucht eines „Fehltrittes“ der Mutter, die ihre Tochter freilich lebenslang für diesen Fauxpas büßen lässt und sie zu sexuellem Verzicht auf Liebe zwingt. Kein Wunder also, dass die schöne Atala keine List mehr weiß, um ihrer Lust teilhaftig zu werden, so dass sie letztlich unter der Last des Christentums zusammenbricht, buchstäblich implodiert.

Seit jeher gab es in der abendländischen Kultur einen Bezug zwischen der Melancholie und dem Kosmos, galt doch der Planet Saturn - den wir bei Bernardin

7 Vgl. Kristeva, Julia: Soleil noir. Dépression et mélancolie. Paris: Gallimard 1987. 
de Saint-Pierre auf der Suche nach seinen kosmischen Fiktionen bereits besucht haben - als Planet der Melancholie schlechthin. Susan Sontag hat sich dieser Konstellation in ihrem oben angeführten Buch Under the sign of Saturn angenähert und kam zu zahlreichen und für unsere Vorlesung wichtigen Erkenntnissen. Sie beschäftigte sich in diesem lesenswerten Band ausgehend von Walter Benjamin und Photographien, die den großen Essayisten und Theoretiker mit auf die Hand aufgestütztem Kopf zeigen, mit verschiedenen kulturellen und literarischen Äußerungs- und Ausgestaltungsformen. In gewisser Weise könnten wir sagen, dass diese Literatur eine Literatur im Zeichen des Saturn ist, vielleicht auch, weil mythologisch gewendet Saturn - wie die Revolution - seine eigenen Kinder frisst.

Depression ist für Julia Kristeva schlicht „le sacre moderne“, wie sie in einem Interview formulierte - und damit ergibt sich erneut eine Beziehung gerade zur Romantik und vor allem zum Schreiben. Denn diese Zeit des „sacre moderne“ ist auch die Zeit des „sacre de l'écrivain“, des unbestrittenen Aufstiegs des Schriftstellers und der Schriftstellerin zu gänzlich herausgehobenen, bisweilen sakralisierten, bisweilen aber auch verdammten Figuren. Die Melancholie umgibt das Schreiben und die Schriftstellerin beziehungsweise den Schriftsteller wie eine Logosphäre, also parallel zur Atmosphäre wie eine unmittelbar um uns befindliche Sphäre, in der wir nicht Luft, sondern Worte, die um uns gesprochen, geschrieben und verbreitet werden, mit allen Sinnen einatmen. Die seit dem ausgehenden 18. Jahrhundert beständig und bedürftig eingeatmeten Bestandteile dieser Logosphäre konfigurieren das „mal de siècle“, eine generationentypische Melancholie, die sich wie eine Epidemie über weite und nicht nur literarische Landstriche auf beiden Seiten des Atlantiks ausbreitete.

In einer Vielzahl von Studien wurde darauf hingewiesen, dass für die Heilkunst der griechisch-römischen Antike die Melancholie mit der Milz verknüpft war, jenem Organ, das in der Medizin unserer Tage justament mit unserem Immunsystem verbunden ist. In gewisser Weise lässt sich die Melancholie - und damit überspannt man meiner Ansicht nach keineswegs den Bogen - als eine Erkrankung des Immunsystems verstehen, folglich als eine Immunschwäche, welche die Widerstandskräfte des Körpers wie der Seele angreift. In der Tat beinhaltet die Melancholie eine ganz grundlegende Frage nach der Beziehung zwischen Individuum und Gesellschaft, wie dies Wolf Lepenies aus Sicht der Kultursoziologie betonte. Wir dürfen mit guten Gründen diese Krankheit sehr wohl in ihren Begründungsstrukturen wie in ihrer spezifischen Symptomatik als Immunschwäche bezeichnen.

Die Briten nennen dieses oft unterschätzte Organ schlicht „spleen“ - der Spleen ist also die Milz. Der Schweizer Literaturtheoretiker Jean Starobinski wies zurecht darauf hin, dass sich die Melancholie schon zu Homers Zeiten symptomatisch als Kummer, Einsamkeit, Verweigerung jeglichen menschlichen Kontakts und ruhelo- 
ses Umherirren äußerte. Aus einer solchen Perspektive erscheint auch der „solitaire“, der christliche Einsiedler und Mönch, als ein Melancholiker, der es geschafft hat, seine eigene Melancholie durch das aktive Handeln für und durch das Christentum zu überwinden und fruchtbar werden zu lassen. Auf ähnliche Weise kann es auch die Schriftstellerin beziehungsweise der Schriftsteller schaffen, aus der Melancholie einen wirksamen Antrieb zu künstlerischer Kreativität zu gewinnen.

Eine besondere Beziehung besteht zwischen Melancholie, Vergangenheit und ihrer Wahrnehmung durch das moderne Subjekt. In gewisser Weise lebt der Melancholiker in der Vergangenheit, so wie Atala letztlich von dem ihrer Mutter gegebenen Schwur nie ganz loskommt. Ähnliches können wir in Paul et Virginie konstatieren: Denn nach Virginies Abreise in Richtung Frankreich wird Paul immer wieder die gemeinsam mit ihr verbrachte Zeit auf der Insel evozieren und nach ihrem Tod sich gänzlich auf ein Denken und Leben in dieser Vergangenheit versteifen. Er hat aufgehört, Pläne für die Zukunft zu schmieden und das Prospektive in der Gegenwart zu kultivieren: Sein ganzes Da-Sein verkümmert zu einem Leben im Vergangenen.

Diese Konstellation mag gleichzeitig auf einen besonderen Bezug der Melancholie zum autobiographischen Schreiben aufmerksam machen, auf eine selbstbezogene Rückwärtsgewandtheit, die das eigene Ich in immer neuen Spiegelungen und Selbstreflexionen gewahr werden und erleben will und weniger an einer Zukunft, als an der Gestaltung von Vergangenheit ausgerichtet ist. Man könnte Roland Barthes' erwähnten Gedanken insoweit ergänzen und weiterführen, als dass angesichts der Melancholie eines Jean-Jacques Rousseau und seiner Rêveries d'un promeneur solitaire nun aus anderen Gründen wiederum Voltaire als der letzte der glücklichen Schriftsteller bezeichnet werden muss. So lässt sich der schwarze Faden der Melancholie bis zurück zu Rousseau verfolgen und überdies eine grundsätzliche Verbindung herstellen mit einem weiten Fächer an autobiographischen Schreibformen, der sich im 19. Jahrhundert kreativ öffnen sollte und auf dessen Wirkungen wir noch vielfach zu sprechen kommen werden.

In diesem Zusammenhang, aber auch mit Blick auf Chateaubriands Atala ist es recht aufschlussreich, nach der Problematik der Sprache und der sprachlichen Gestaltung sowie nach deren Grenzen zu fragen. Gibt es nicht Bereiche, in welche das moderne Subjekt mit seiner ausgefeilten und an den Gegenständen ausgerichteten Sprache nicht mehr vordringen kann, die nicht mehr erreichbar scheinen, die folglich nicht mehr literarisch oder psychoanalytisch bearbeitbar sind? Gewiss würde ein Psychoanalytiker - insbesondere aus der Schule von Jacques Lacan es jederzeit verneinen, dass diese Unmöglichkeit, mit der Sprache in die verstecktesten und tiefsten Bereiche der menschlichen Psyche vorzudringen, überhaupt bestehen könnte. Aber denken wir an Roland Barthes' letzten zu Lebzeiten veröffentlichten Band, nehmen wir also das Beispiel von La Chambre claire, so ließe 
sich schon fragen, ob in dieser Note sur la photographie ${ }^{8}$ im Zurückdenken an die verstorbene, verschwundene Mutter nicht ein abwesendes Bild an die Stelle der geliebten Henriette Barthes getreten ist. Mit anderen Worten: Es eröffnet sich gerade im melancholischen Rückblick des Zeichentheoretikers auf die gemeinsame Zeit langer Jahre des Zusammenlebens mit seiner Mutter ein Bereich, der nicht mehr anders als in Bildern - und selbst mit Hilfe eines Bildes, das als einziges im Band nicht abgebildet ist - gesagt werden kann. Dies bedeutet nicht, dass die Grenzen der Sprachen eingestanden würden, aber sehr wohl, dass sie zu einem Gegenstand der Selbstreflexion geworden sind.

Dieses (momentan oder letztlich) Unsagbare hat zweifellos mit der Melancholie, der Depression, der schwarzen Sonne der Künstlerin beziehungsweise des Künstlers zu tun. Denn die Kunst, die Literatur, tritt gerade dort in ihr Recht, wo sie an den Grenzen des Sagbaren, des Auszudrückenden arbeiten kann und ihre ganze Kreativität in die Waagschale werfen muss, um dem scheinbar Nicht-Sagbaren, dem Nicht-Ausdrückbaren Worte und Sätze abzuringen. An dieser Stelle könnten wir einige Überlegungen Julia Kristevas einbinden, die sich gerade der Problematik des Sprechens und der Muttersprache widmen:

Der spektakuläre Zusammenbruch des Sinns beim Depressiven - und im Extremfall des Sinns des Lebens - lässt uns folglich annehmen, dass es ihm Schwierigkeiten bereitet, die universelle Bedeutungskette, also die Sprache, zu integrieren. Im Idealfalle bildet das sprechende Subjekt mit seinem Diskurs ein Ganzes: Ist das Wort nicht unsere „zweite Natur“? Im Gegensatz dazu ist das Sagen des Depressiven für diesen wie eine fremde Haut: Der Melancholiker ist ein Fremder in seiner eigenen Muttersprache. Er hat den Sinn - den Wert - seiner Muttersprache verloren, in Ermangelung des Verlusts seiner Mutter. Die tote Sprache, die er spricht und die seinen Selbstmord ankündigt, verbirgt eine Sache, die lebendig begraben wurde. Aber er wird sie nicht übersetzen, um sie nicht zu verraten: Sie wird in der „Krypta“ des unsagbaren Affekts eingemauert, anal gefangen, ohne Ausweg. ${ }^{9}$

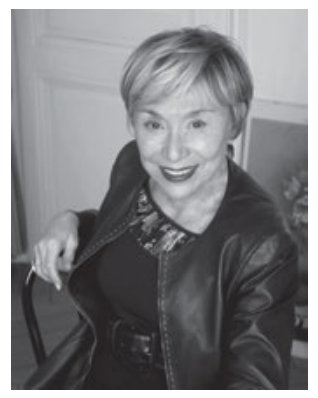

Abb. 21: Julia Kristeva (Sliwen, Bulgarien, 1941) im Jahr 2008.

8 Vgl. Barthes, Roland: La Chambre claire. Note sur la photographie. Paris: Cahiers du Cinèma Gallimard - Seuil 1980.

9 Kristeva, Julia: Soleil noir: dépression et mélancolie, S. 64. 
Die Sprache, ja mehr noch die Muttersprache, welche die zweite Natur des Menschen ausmacht, wird hier dem Subjekt zum Ausdruck des Sich-selbst-fremdSeins. Diese Muttersprache ist dem depressiven Menschen plötzlich nicht mehr natürlich, nicht länger ein selbstverständliches Ausdrucksmittel seines Wesens, seines Seins. Die von ihm gesprochene Sprache ist Ausdruck des Nicht-mehr-Sagbaren, die Muttersprache geht ihm gleichsam in ihrer Sinnhaftigkeit verloren, wird für den Depressiven zu einer Fremd-Sprache des Eigenen, zum Ausdruck des Fremden im Eigenen.

Damit aber wird das unter Depressionen leidende Subjekt, also der Sprecher oder die Sprecherin auf sich selbst zurückgeworfen, verständigt sich nicht mehr mit dem anderen, mit der Gemeinschaft, mit der Gesellschaft. Atala wird dies ebenso tun wie René, der andere große melancholische Held und das männliche Gegenstück Atalas in Chateaubriands romanesker Schöpfung. Nicht anders in Paul et Virginie: Paul wird in der Präsenz wie später in der Absenz seiner Mutter kaum mehr die Möglichkeit zu muttersprachlicher Kommunikation haben. Die Melancholie bedingt damit eine fundamentale Sprachstörung, eine diskursive Ver-Störung, welche letztlich zur Zer-Störung des Subjekts führt und in der sich das Ich selbst zum Fremden oder zur Fremden wird. Bernardin de Saint-Pierre ist in der Tat in vielerlei Hinsicht ein Vorläufer, der in seinem literarischen Schaffen jene Ver-Störung bearbeitete, welche - denken wir an die Generation der Werther-Leser - zur Selbst-Zerstörung zahlreicher junger und einst hoffnungsvoller Melancholiker führte.

Fehlende Kommunikation treibt bei Chateaubriand die schöne Atala zum Selbstmord; doch ist ihr die Muttersprache selbst fremd geworden, gerade ihr als biologischer Mestizin, die zusätzlich zu der von Kristeva konstatierten Fremdwerdung und problematischen Mutterbeziehung auch sich selbst als Fremde, als weder den einen - den Weißen - noch den anderen - den Indianern - zuordenbar erlebt. Wie Kleists Toni ist sie im Zwischenraum des Weder-Noch zum Tode, zum Nicht-länger-leben-Können verurteilt. In diese klaffende Lücke, in diese Sprachlosigkeit aber begibt sich auf den Spuren Bernardin de Saint-Pierres die Kunst eines Chateaubriand. Und wir könnten hinzusetzen: ein gut Teil der romantischen Kunst, die auf beiden Seiten des Atlantiks ihre zum damaligen Zeitpunkt innovativen Kreationen weiterspinnt.

Depression als entweichende Pression, als weichender Druck im Innern des Ich, führt gleichsam zu einer Implosion des Ich, das sich nicht mehr äußern, also nach außen treten und mit anderen Menschen kommunizieren kann. So bleibt dieses Ich - um Julia Kristevas Metapher aufzunehmen - in seiner eigenen Krypta eingemauert, also von allen anderen getrennt in einem Reich des Todes, und mehr noch: lebendig begraben, nach innen gekehrt. Diese Implosion des Ich wird nur auf einer einzigen Ebene überwunden: in der Sprache der Kunst, in der Sprache 
der Literatur. Es geht in diesem fatalen Zusammenhang weniger um eine Kunst des Sprechens, sondern um eine Kunst des Schreibens, dessen therapeutische Wirkung damit offenkundig wird. So besteht also eine fundamentale Beziehung zwischen der Selbstreflexivität beziehungsweise Selbstbezogenheit des Melancholikers, zwischen seiner Sprachstörung und den Tendenzen zur Selbstzerstörung sowie schließlich den Möglichkeiten eines literarischen Schreibens, das seine heilenden Wirkungen entfalten kann.

Bereits seit der abendländischen Antike erscheint die Melancholie als eine den Menschen und dessen Leben gefährdende Krankheit. Sie ist schon bei Hippokrates mit der Beobachtung verbunden, dass wenn gemäß der antiken Säfte-Lehre Feuchte und Traurigkeit lange anhalten, der Zustand der Melancholie vorliege. Eine grundlegende Beziehung der Melancholie bestehe überdies auch zur Galle, deren schwarze Farbe dieses Organ wohl in besonderer Weise mit den schwarzen Ideen der Melancholiker verknüpfe. Die Antike sah folglich eine Beziehung der Krankheit Melancholie mit den vier Qualitäten trocken, feucht, warm und kalt einerseits und den vier Elementen Wasser, Luft, Erde und Feuer. Gemäß dieser Lehre wurde die Melancholie mit der Erde verbunden, die trocken und kalt ist; aber auch mit dem Herbst, jener Jahreszeit, in der die Galle ihre stärkste Kraft entfaltet.

Bemerkenswert und für unsere Überlegungen wichtig ist Jean Starobinskis Hinweis, dass das Adjektiv „melancholos“ auch im Sinne einer Vergiftung und eines Giftes verwendet wurde, in das Herakles seine Pfeile getaucht habe: in das todbringende Gift der vielköpfigen Hydra. Erneut sehen wir in diesem Zusammenhang also die schillernde Bedeutung des Pharmakons aufscheinen. Die einmal erworbene Krankheit, so stellte Starobinski ferner fest, könne sich direkt auf den Körper beziehen, wobei sie zu Epilepsien führe, oder aber auf Geist und Intelligenz, wodurch sie definitiv zur Melancholie werde. Nach einer bis ins 18. Jahrhundert hinein vorherrschenden Theorie handelte es sich bei der Melancholie um eine schwere Erkrankung der schwarzen Galle, wobei das vergiftete Blut entweder nur den Kopf oder aber den gesamten Körper erreiche. Sie sehen, unsere schöne Atala brauchte eigentlich gar kein Gift: Ihr Körper hatte es gleichsam in sich gespeichert und einen Tod programmiert, der alles vergiftend von innen kommt! Nach einer seit der Antike kolportierten Theorie handelte es sich letztlich um schwarze Dämpfe, die im Körper der Erkrankten aufsteigen und nicht allein schwarze Ideen, sondern auch bestimmte Halluzinationen hervorrufen konnten. Sowohl Bernardin de Saint-Pierre als auch François-René de Chateaubriand konnten folglich auf ein reiches Arsenal an Symptomatiken und deren Erklärungen zurückgreifen, was sie bei der Gestaltung ihrer pathologisch affinen Figuren auch taten.

Die Melancholie der Moderne ist ein freilich weniger stark im Medizinischen und Pathologischen verankerter Diskurs. Nicht selten wird sie mit kulturhistorischen Aspekten verbunden, die eine bislang noch nicht von uns reflektierte 
Zeitlichkeit in diesen Diskurs über Melancholie in der Sattelzeit der Romantik bringen. Die Berücksichtigung von Zeitlichkeit aber ist wichtig, um bereits die frühen Äußerungsformen einer derartigen Temporalisierung im Kontext einer Historisierung aller Lebensbezüge ab dem Ende des 18. Jahrhunderts besser zu verstehen. In einem solchen Kontext leitet sich die Melancholie von einem Zuspät-Kommen - oder bisweilen auch Zu-früh-Kommen - in einem zu modernen oder zu alten Jahrhundert her. Der Melancholiker hat folglich seine Zeitepoche nicht getroffen, sondern deutlich verfehlt: Er oder sie trauert zu Zeiten der Revolution einem verflossenen Ancien Régime nach oder lebt in einer von aufkommenden Nationalismen zerrissenen Welt in Sehnsucht nach einer Welt in Frieden und Differenz. Beide Situationen problematischer Zeitlichkeit und Eigenzeitlichkeit sind für das Subjekt nicht ohne weiteres lösbar.

Es gibt mithin so etwas wie einen chronologischen Sprung, einen Abgrund, der nicht überbrückt werden kann. Gewiss ist dies im absoluten Sinne kein für die Moderne oder gar die Romantik spezifischer Zug, denken Sie nur an Miguel de Cervantes' Don Quijote, William Shakespeares Hamlet oder auch an Richard Burtons Anatomy of Melancholy aus dem frühen 17. Jahrhundert! Für sie alle könnte Shakespeares Vers sprechen: „Time is out of joint“. Und es gibt für den Melancholiker oder die Melancholikerin nichts und niemand, der diese aus den Fugen geratene Zeit wieder dauerhaft kitten könnte.

Bei Jean-François de Saint-Lambert, also beim literaturgeschichtlichen Übergang von der Aufklärung zu einer Genieästhetik, findet sich der Schriftsteller, der Dichter, bereits in Begleitung der schönen Dame Melancholie. Die Verbindung von Melancholie und Weiblichkeit ist dabei traditionell in den abendländischen Geschlechterrollen angelegt, da die Frau insgesamt als weniger aktiv und schwächer angesehen wird, so dass sie leichter von der Melancholie heimgesucht werden und ihre Lebenslust verlieren kann. Es gibt noch eine weitere Zweiteilung, die von Madame de Staël in ihrem Werk De l'Allemagne eingeführt wurde, mit dem wir uns später noch in unserer Vorlesung beschäftigen werden. Bei ihr erhält die Melancholie eine positive Wendung, denn sie wird zum Symbol der von ihr so apostrophierten Literaturen des Nordens. Dagegen seien die Literaturen des Südens seit der Antike konkret, hell und heidnisch, während die des Nordens träumerisch, von Nebelschwaden umwölkt und ideell seien. Mit dieser Wendung vollzieht sich eine Realisierung und Anpassung der Klimatheorien des 18. Jahrhunderts, wie sie etwa von Montesquieu und Voltaire vorgetragen worden waren. Von einem so umrissenen Punkt aus gibt es eine Beziehung zu Chateaubriands Scheidung zwischen antiker Exteriorität und moderner Interiorität, wobei er letztere im Zeichen des Christentums als überlegen deutete. Sie sehen, die jeweils sehr komplexen Verästelungen der Melancholie beziehungsweise des Melancholischen wären eine eigene Vorlesung wert! 
Julia Kristeva weist uns in Soleil noir mit ihrer Theorie darauf hin, dass die nicht gelöste Mutterbeziehung von ganz zentraler Bedeutung für die Melancholie, aber auch für die damit verbundene Sprachstörung ist. Die eigene Sprache wird dem Subjekt fremd, sie führt zu einer Störung der Beziehungen zur Außenwelt, zu einer Verstörung mit Blick auf die Selbstbefindlichkeiten des Ich und in letzter Konsequenz zu einer Zerstörung und Selbstzerstörung dieses Subjekts, das sich in seinem ureigensten Bereich lebendig einmauert. Im nachfolgenden Zitat von Sören Kierkegaard wird sich zeigen, wie die Langeweile im Sinne der Melancholie in eine Problematik der Sprache übergeht - und der Begriff der Langeweile bildete sich erst in der zweiten Hälfte des 18. Jahrhunderts heraus. Diese Problematik der Sprachstörung führt uns fast notwendig zum Turmbau zu Babel, der letztlich für diese Elemente einsteht: das Fremdwerden gegenüber der eigenen Sprache, die Problematik der abreißenden gemeinschaftlichen Kommunikation, die individuelle Vereinzelung und schließlich auch die melancholische Rückwendung $\mathrm{zu}$ einer verlorenen gemeinsamen Sprache, einer verlorenen Einheit der Menschheit ${ }^{10}$ im sprachlichen Sinne.

Chateaubriands Atala jedenfalls verkörpert ganz ohne jeden Zweifel diese grundlegende Sprachstörung, so wie wir bereits die Sklavin Marie in Bernardin de Saint-Pierres Paul et Virginie stumm auf ihrem Felsen sitzen sahen, unfähig dazu, ihre Trauer in Worte zu kleiden. Immerhin wird sie sich im Gegensatz zu Atala, aber indirekt auch zu Paul nicht selbst umbringen. Ganz allgemein gesprochen ist der Melancholiker folglich jener Mensch, dem es nicht gelingt, die Kommunikation mit anderen durch die gesprochene Sprache wiederherzustellen. Der Verlust der Sprache ist - ganz wie die aufsteigenden schwarzen Dämpfe - ein Gift, das von innen kommt und nicht von außen auf die Opfer einwirkt.

Noch ein letztes Wort zur Körperhaltung des Melancholikers, der seinen Kopf auf die eine Hand stützt beziehungsweise seinen Kopf in der einen Hand hält! Diese Haltung ist uns allen aus Albrecht Dürers Melancholie vertraut. Susan Sontag hat sie in ihrem Buch Under the Sign of Saturn wiedergefunden: bei Walter Benjamin, dessen Photographien ihn immer wieder in dieser Position zeigen. Das kann bei einem so präzise sprachlich inszenierenden Schriftsteller und Kritiker wie Benjamin kein Zufall sein. Bei ihm aber ist die Melancholie zu einer Art Antriebskraft, zu einer dynamischen Schaffenskraft geworden, die wir im Folgenden noch etwas näher ergründen wollen.

10 Vgl. hierzu auch Olender, Maurice: Die Sprachen des Paradieses. Religion, Rassentheorie und Textkultur. Revidierte Neuausgabe. Herausgegeben und mit einem Vorwort von Markus Messling und einem Vorwort zur Erstausgabe von Jean-Pierre Vernant. Mit einem Essay von Jean Starobinski. Aus dem Französischen von Peter D. Krumme. Berlin: Kulturverlag Kadmos 2013. 
So freilich kann der „furor melancolicus“ zunehmend den „furor divinus“ ersetzen: Die Melancholie wird nach der Jahrhundertwende immer mehr zu einer spezifischen Eigenschaft, einem Ausweis des Künstlers. Sie kann dabei nicht nur als erlittene, sondern auch als erwählte Krankheit auftreten - eine durchaus wichtige Veränderung gerade auch mit Blick auf die Romantik und das gesamte 19. Jahrhundert. Die Melancholie geht damit ein in den Habitus des Künstlers, eine Tatsache, die gerade bei der bildhaften Repräsentation der Literat*innen und Künstler ${ }^{\star}$ innen des 19. Jahrhunderts - und bis weit ins 20. Jahrhundert hinein eine wichtige Rolle spielte. Wir sehen in diesem sich langsam vollziehenden Prozess, wie die Melancholie von den Figuren der Literatur auf die Verfertiger von Literatur überspringt und sie gleichsam als von der Melancholie getriebene sensiblere, empfindsamere Menschen adelt.

Melancholie kann in der Spielart der Langeweile, des später so berühmten „ennui“, auch in einem ganz existential-philosophischen Sinne zu einem Motor der Geschichte schlechthin werden. Wie sehr sich die Melancholie im Gewand der Langeweile an der Wende zum 19. Jahrhundert bereits ausgebreitet hatte, lässt Johann Gottlieb Fichte in seinen philosophischen Überlegungen ab der Jahrhundertwende zunehmend deutlicher werden. Er war zuvor ein Verteidiger des europäischen Kosmopolitismus gewesen, schwenkte dann jedoch unter dem Einfluss der französischen Hegemonie im Zeichen Napoleons um, radikalisierte sich zunehmend und wurde spätestens mit seinen 1807 und 1808 zunächst als Vorlesungen gehaltenen Reden an die deutsche Nation im damals französisch besetzten Berlin zu einem Verteidiger der nationalen (und letztlich nationalistischen) Sache. Doch schon in seinen Äußerungen über den geschlossenen Handelsstaat schrieb Fichte im Jahre 1800: „Zu reisen hat aus einem geschloßnen Handelsstaate nur der Gelehrte und der höhere Künstler: der müßigen Neugier und Zerstreuungssucht soll es nicht länger erlaubt sein, ihre Langeweile durch alle Länder herumzutragen.“

Interessanter aber noch ist vielleicht der Ansatz Sören Kierkegaards, der aus existential-philosophischer Sicht die Langeweile zum roten Faden der Menschheitsgeschichte machte. Gleichsam ein „Je m'ennuie, donc je suis“ - ich langweile mich, also bin ich. Die Langeweile kann als Spielart der Melancholie eine vorwärts treibende kreative Kraft sein, gerade im Kontext des Gefühls, in einer falschen Zeit, zu früh oder zu spät, leben zu müssen. Sie kann also der Begriff für Lebensverdrossenheit, ja einer Lebensmüdigkeit sein, welcher der dänische Philosoph in seinen Überlegungen unter dem Titel Entweder - Oder ganz neue Seiten abgewann: 
Die Götter langweilten sich, darum schufen sie die Menschen. Adam langweilte sich, weil er allein war, darum ward Eva erschaffen. Von diesem Augenblick an kam die Langeweile in die Welt, wuchs an Größe in genauer Entsprechung zum Wachstum der Menge des Volks. Adam langweilte sich allein, alsdann langweilten Adam und Eva sich im Verein, alsdann langweilten Adam und Eva und Kain und Abel sich im Familienkreis (en famille), alsdann nahm die Menge des Volks in der Welt zu und langweilte sich en masse. Um sich zu zerstreuen, kamen sie auf den Gedanken, einen Turm zu bauen, der so hoch sei, dass er emporragte in den Himmel. Dieser Gedanke ist eben so langweilig wie der Turm hoch war, und ein erschrecklicher Beweis dafür, wie sehr die Langeweile überhand genommen hatte. ${ }^{11}$

Soll ich an dieser Stelle der Vorlesung nun hoffen, dass Sie sich vorzüglich langweilen? In jedem Falle haben Sie in dieser Passage eine Universalgeschichte der Langeweile vor sich, die letztlich zur Entfaltung aller kreativen Kräfte der Menschheit führte. Im Grunde lässt sich in diesen geschickt gestaffelten Sätzen zum einen die zerstörerische, aber auch die gestaltende Kraft von Langeweile und in letzter Konsequenz ebenso der Melancholie aufzeigen. Denn es ist die nicht mehr weiter hinterfragbare Lebensmüdigkeit, die die Aktivitäten entfaltet, weil man mit dem, was die anderen Menschen so treiben, auf keinen Fall konform gehen kann.

Das moderne Subjekt, das hatten wir seit Jean-Jacques Rousseau gesehen, ist ein Subjekt, das sich aus der Trennung von den Anderen heraus begreift und sich an Dingen erprobt, die nicht notwendigerweise in einem utilitaristischen, unmittelbar zweckgebundenen Zusammenhang stehen. Mit dem Bungee-Springen verfolgt es kein utilitaristisches Prinzip; es sei denn, Sie betrachten den Veranstalter des Events, der seine Frau und die sieben Kinder davon ernähren muss. Die Melancholie ist daher ein grundlegend ambivalentes Phänomen: Sie kann auf eine ständige Rückwendung zur eigenen Vergangenheit hinauslaufen, in einem fast absoluten Sinne handlungs- und veränderungshemmend sein, den ganzen Körper und Geist lähmen und das Gefühl absoluter Vereinzelung mit sich bringen. Doch kann sie auch in Aktivität umschlagen, ja in eine nicht mehr abreißende Abfolge von Aktivitäten, gerade um dieses Gefühl der Langeweile aus dem Wahrnehmungsbereich zu tilgen und auszuschalten. Denn Fingernägelkauen alleine genügte schon Adam und Eva nicht.

Fassen wir die Melancholie - immer Walter Benjamins Bild vor Augen - nicht zuletzt als menschliche Produktivkraft auf! In der Melancholie ergibt sich eine radikale Auseinandersetzung des Subjekts mit seinem Ursprung und seinen Zielen in der Welt. Eben dies führt zu einer doppelten Bewegung ins Innere und in die äußere Welt, insbesondere auch im Sinne der Reisen durch sie. Vergessen wir dabei Fichtes obige Mahnung nicht, Reisewarnungen nicht wegen bestimmter

11 Kierkegaard, Sören: Entweder - Oder. Erster Teil. Düsseldorf: Diederichs 1956, S. 305. 
Infektionsgefahren, sondern wegen der Ansteckung mit Langeweile auszusprechen! Sie merken, es gibt Epidemien, die weder rein positiv noch rein negativ zu bewerten sind: Die Epidemien der Melancholie und der Langeweile gehören dazu.

Als Paul seine Virginie in Bernardin de Saint-Pierres Roman verliert, entgleitet ihm auch der Halt in einem Raum, der ihm einst so vertraut, so lieb und teuer war - er irrt ziellos umher. Atala sucht einen Weg aus dem Indianerlager, als sie die Möglichkeit erkennt, mit Chactas' Hilfe aus der ihr fremd gewordenen Welt des Eigenen auszubrechen. René selbst hat in Atala seinem geliebten Frankreich den Rücken gekehrt, um sich in der Weite des Raumes zu verlieren und sich selbst vergessen zu können. Was zählt da noch die Suche nach der Nordwest-Passage! Handelte Christoph Kolumbus, als er sich auf den Weg nach Osten über den Westen machte, vielleicht doch nur aus Langeweile? Wir müssen dieses Phänomen keinesfalls als rein selbstzerstörerisch oder rein schöpferisch, sondern in seiner ganzen Ambivalenz erfassen und begreifen.

Es gibt sie daher, die „Räume der Langeweile und Melancholie“, von denen Wolf Lepenies in seiner angeführten Studie sprach, ${ }^{12}$ auch wenn ich hier seinem kultursoziologischen Spürsinn nicht unbedingt folgen mag. Immer wieder aber finden wir, dass sich Melancholie und Langeweile letztlich an Zivilisationsgrenzen abarbeiten, an Grenzen der Zivilisation entweder des Eigenen oder des Fremden, und dass letztlich kraft der Melancholie auch die Position der eigenen Zivilisation produktiv in Frage gestellt wird. Der Lebensmüdigkeit tritt oft die Zivilisationsmüdigkeit an die Seite. Daher auch die nicht nur bei Bernardin de SaintPierre und viel stärker noch bei Chateaubriand oder dem jungen Victor Hugo in Bug-Jargal - mit diesem Roman beschäftigen wir uns gleich - zu beobachtende implizite oder auch explizite Zivilisationskritik, die freilich politisch sehr unterschiedlich verankert sein kann.

Es ist bemerkenswert, dass Johann Gottlieb Fichte gerade den reisenden Müßiggängern das Reisen verbieten wollte, weil sie mit ihrer Melancholie und ihrem Geld diese Krankheit wie sich selbst nur in andere Länder schleppten und andere damit ansteckten. Melancholie und Reise stehen in einem eigentümlichen Verhältnis zueinander: Es ist eine Krankheit der Romantik und der Romantiker, diese äußerste Gespanntheit und Ich-Versunkenheit, diese extreme Aktivität und neurotische Hypersensibilität, welche die Romantik in Europa mit der Romantik in anderen Teilen unseres Planeten verbinden. Ich möchte diesem Weg nun im weiteren Verlauf der Vorlesung etwas folgen und sehen, in welchem Maße sich bestimmte Konstellationen, die wir bislang in der französischen Literatur näher betrachtet haben, sich auch in Übersee auffinden lassen.

12 Vgl. Lepenies, Wolf: Melancholie und Gesellschaft, S. $115 \mathrm{ff}$. 
Dabei soll untersucht werden, welche Funktionen diesen Elementen oder Komponenten in einem veränderten Kontext zukommen und auf welche Weise sie jeweils umfunktionalisiert, also mit neuen Funktionszuschreibungen ausgerüstet werden. Denn es ist etwas anderes, ob wir die romantischen Utopien, Heterotopien oder Traumorte in die Ferne projizieren und von Europa aus an Orte denken, die zu Projektionsflächen europäischer Vorstellungen geworden sind, oder ob wir von Räumen und Regionen aus schreiben, für welche europäische Imaginationen, Bilder und Konzepte zum Teil gänzlich andere Funktionen vor Ort übernehmen. Doch bevor wir in unserer Vorlesung nach Lateinamerika springen und einen fundamentalen Blickwechsel vollziehen, möchte ich Ihnen noch ein letztes Mal einen europäischen Text vor Augen führen, der im Zeichen romantischer Figurationen einen außereuropäischen und zugleich amerikanischen Schauplatz wählt, den wir bereits gut kennen. 